Haya: The Saudi Journal of Life Sciences

Abbreviated Key Title: Haya Saudi J Life Sci

ISSN 2415-623X (Print) |ISSN 2415-6221 (Online)

Scholars Middle East Publishers, Dubai, United Arab Emirates

Journal homepage: https://saudijournals.com

Review Article

\title{
Versatile Fusion of Renown Pediculicidal Agents: An Innovative Thought
} Dr. Asma Naeem ${ }^{1 *}$, Anila Shehzadi ${ }^{2}$

${ }^{1}$ Department of Zoology, Faculty of Science, The University of Central Punjab, Pakistan

${ }^{2}$ Department of Zoology, The University of Central Punjab, Pakistan

DOI: 1 10.36348/sjls.2021.v06i03.001 | Received: 25.02.2021 | Accepted: 09.03.2021 | Published: 10.03 .2021

*Corresponding author: Dr. Asma Naeem

\section{Abstract}

Pediculus humanus has always been the most obstinate ectoparasites that evolved with human beings and persist till today throughout the world. These wingless nemeses are extremely hard to deal with, because they prefer the human body temperature for their existence and propagation or reproduction. Numerous studies have been conducted to exterminate these blood feeders by the employment of several natural as well as synthetic regimens but not with an ideal proficiency. Anyhow, a multifaceted product would be beneficial for killing and removing the lice and their stubborn nits from the human hair shafts permanently. Our current focus is to introduce an all rounder treatment with a marvellous fusion of deltamethrin (DMT) and diisopropylfluorophosphate (DFP) along with dimeticone (PDMS). The tonic lotion with the amalgamation of aforementioned antilice candidates will stop lice reproduction, accessory gland secretions that are mediated by corpora allata, nit formation, neurotransmission and behavioural responses which will ultimately lead to clearance of these hematophagous insects. So as there is no promising solution to this global discomfort at present, our novel proposal will hopefully pave a new way to eradicate lice forever.

Keywords: Hematophagous, DMT, DFP, PDMS, nit removal, lice killing, accessory gland secretions, nerve transmission.

Copyright ( $) 2021$ The Author(s): This is an open-access article distributed under the terms of the Creative Commons Attribution 4.0 International License (CC BY-NC 4.0) which permits unrestricted use, distribution, and reproduction in any medium for non-commercial use provided the original author and source are credited.

\section{INTRODUCTION}

Multiple steps are being taken for several years to get rid of a highly transmissible and historic epizootic menace due to Pediculus humanus (holding both species as human head and body lice) all over the world [1]. Pediculus humanus (lice) entirely depends upon the human blood meal which may lead to iron deficiency, impotence of the hemoglobin to carry oxygen into the circulatory system and anaemia in the affected person [2]. Lice tend to inject their digestive juices as well as waste nitrogenous material into the skin/scalp while piercing for sucking blood. All of these lice by products cause severe itching that becomes annoying and embarrassing at times for a person having lice. Moreover, human lice can spread diseases such as typhus, relapsing fever and trench fever [3], the adult female louse can survive for a month and lays approximately up to ten eggs per day that get tightly sticked to their host hair shaf, known as "nits", also referred as fertilized eggs of the sucking louse [4] and empty eggshell of louse [5] which are extremely hard to be removed because in contrast with the adult lice, the eggs of human lice contain an impervious hydrophobic (water hater) cuticle, making them waterproof, that is the reason why these nits are not easily exterminated with water simply [6]. These nits/eggs take about a week to hatch and thus transform into an adult in the other week [7]. Albeit Pediculus humanus is an extraordinarily tiny hexapod, it carries all the biological systems as well as mechanisms involved for sustenance like other insects. Allatotropins (AT) are insect neuropeptides (small proteins produced by nerve cells) and are versatile in their action and perform a lot of functions in different insects such as impedance of ionic exchange in the midgut, facilitating the peristaltic movements in the foregut, and hastening of the heart rate etc [8]. These neuropeptides interact with the $G$ protein coupled receptors (GPCRs) or seven transmembrane domain receptors (7TM), which communicate with the $G$ protein inside the cell membrane, providing specific target sites for approximately fifty percent of the drugs as well as insecticides for binding, as it is involved in several physiological activities in insects including growth, reproduction, and development. These receptor proteins also act as chemosensory receptors [9] and signal 
transducers, because they receive the extracellular information and initiate a cascade of proteins and protein complexes associated with intracellular responses [10]. In the same manner, they mediate ganglionic nerve (impulse) transmission and also trigger corpora allata. Further, corpora allata (a pair of endocrine glands or secretory cell clusters, located at the anterior end) governs the secretion of two vital hormones as juvenile hormone $(\mathrm{JH})$ also known as "neotenin", and 20 hydroxyecdysone (20E). Both of these hormones ultimately stimulate the production of secretory substances from the accessory glands and are significant for the egg production in the female lice [11]. Moreover, the secretions of male accessory glands (AGs) consist of diversified amino acid sequences in the form of peptides and proteins, carbohydrates and lipids [11], these secretory products induce alterations in the female endocrine system during mating, thus acceleration of oviposition and deceleration of sexual receptivity of the mated female occurs [12]. Various pediculicides have been employed which proved unsuccessful in case of lice eggs' treatment [6]. Some topical anti lice products including natural pyrethrins (which is commonly derived from chrysanthemum), synthetic pyrethroids (e.g., permethrin and deltamethrin) and organophosphates (also known as phosphate esters containing malathion) along with natural herbal oils are considered more promising against Pediculus humanus [1]. Further, pyrethroids are one of the most commonly used pediculicidal agents such as deltamethrin. A recent experimental study has revealed that the sensitive population of salmon lice are affected by deltamethrin which primarily targets the nerve cells and muscle tissues, thus resulting in immobilisation of these lice [13]. According to Bernstein J. E [7], when $0.025 \%$ fluorophosphate (or diisopropylfluorophosphate) was applied to human louse nits obtained from an affected person's hair shaft, and kept under observation for fourteen days, no lice hatched from those nits. Moreover, dimethicone or silicone oil has been pediculicidal claimants as well as discuss the fruitful consequences by the utilisation of their merger.

\section{Accessory glands (AG) and their functions}

The accessory and cement/ collateral glands in the male and female insects respectively, are of great importance in the reproduction. Like other insects, Pediculus humanus capitis (human head louse) also produces a cementing substance by the female cement glands for the protection of their eggs [14] and for their long sticking to the hair shaft, thus preventing them from being discarded [4]. Further, these accessory secretions of the female sex accessory glands (SAGs) are poured into the oviduct before egg laying, thus helping in oviposition i.e., attachment of the eggs to the substrate or hair. At the time of copulation, the spermatophore (containing sticky protective walls) is released from the male accessory glands and transferred to the female reproductive tract [15] which then gets inflated and forces the mature sperm from the spermatophore, when it is encountered with the female collateral secretions.

\section{Composition of the adhesive substances of female AGs}

The female cementing secretions obtained from the nit cylinders were biochemically analysed by the pyrolysis gas chromatography as well as mass spectrometry, which has shown the constituents/ ingredients found in the secretory substances, which include phenyl alanine, tyrosine, tryptophan, glutamic acid [5], major proteinaceous content (as protein or peptide based), consisting of four bands and crosslinking with other aliphatic compounds [14]. These gluey products also contain diversified amino acid sequences along with carbohydrates and lipids [12], aiding in egg production and prevention from remating of the mated female insect [11].

\section{Stimulatory mechanism of the insect AGs}

The accessory glands are vital for the survival of their newly laid eggs in order to sustain their species. The stimulation of these glands is governed by several proteins, hormones and neuropeptides which are discussed below:

\section{Corpora allata (CA) and allatotropins (AT)}

Corpora allata (sing: allatum) is a paired ectodermal organ in the posterior part of all primitive insects' heads (including order phthiraptera) i.e., behind the brain and is a chief source for the production of juvenile hormone $(\mathrm{JH})$ as well as 20 hydroxyecdysone [11], both of which trigger the secretions of insect accessory glands. Additionally, allatotropins (AT) are the peptidic products secreted by neurons which evoke CA to synthesise its hormonal secretions [16], which are considered as the most primitive signaling molecules, serving as potent neurotransmitters [17]. Further, the developmental switching among the hemi and holometabolous insects is entirely based upon $\mathrm{JH}$ titres produced by $\mathrm{CA}$, under the controlling action of AT [18].

\section{Role of GPCRs in AG secretions}

$\mathrm{G}$ protein coupled receptors, also known as seven (alpha helical) transmembrane receptors or 7TM, which belong to the superfamily of transmembrane domain proteins [19] and mediate allatotropins which ultimately accelerate the secretions of the human louse accessory or collateral glands. These receptors get activated by the hydrolysis of GTP, leading to the breakdown of $\mathrm{G}$ protein (linked to these receptors) and hence the intracellular signalling starts [20]. In contrast with other insects, the Pediculus humanus humanus (human body louse) possesses the minimal pool of GPCRs (and 104 nonsensory GPCRs) along with a slighter number of functional odorant binding proteins (OBPs), chemosensory proteins (CSPs), and insulin/target of rapamycin (TOR) signaling pathway, 
well studied in the fat body and CNS of D. melanogaster [21], associated with the nervous system [22] and play a key role in the environmental sensing and responding. Moreover, according to the researchers at Purdue University of West Lafayette reported that GPCRs in human body lice, are responsible for the neurological signaling as well as vision with three opsins (i.e., the vision receptors), thus having a little sight or perception [22]. In agreement with Hill at Purdue University 2010 [23], GPCRs are related to the nervous system of human lice, serving as the most effective target sites for a vast variety of novel insecticides [24].

\section{Deltamethrin (DMT): as an effective pediculicide}

Pyrethrins are of vital significance as pediculicides, obtained from dried flowers of Chrysanthemum cinerariaefolium [25] and are mainly classified into two types as type I pyrethrins and type II pyrethrins [26]. Deltamethrin (DMT) is a synthetic version of the natural pyrethrin, comes under the second category of pyrethrins and is a colourless, odorless cyclopropanecarboxylate ester with a molecular weight of $505.21 \mathrm{~g} / \mathrm{mol}$, existing as a single stereoisomer, having a strong pediculicidal (lethal to lice) effect [27]. DMT is less toxic to human beings [28] because of their higher body temperature, and a little sensitivity towards it, but is definitely lethal to the ectoparasites (with up to 2250X more toxicity) due to their small size, low body temperature as well as an increased sensitivity for DMT [29].

\section{Mechanism of action of DMT}

Deltamethrin incredibly exerts neurotoxic effects by the disruption of sensory and motor neuronal transmission hence thesignalling renders ineffective to the $\mathrm{CA}$, engaged in the production of accessory glands secretions. The presence of an alpha cyano group and phosphorylated configuration in DMT triggers a prolonged inhibition of the sodium channels (also antagonist to calcium channels), preventing them from being activated [30] or may cause the persistent openings of the sodium channels which leads to a continuous membrane depolarisation and recurrent firing of the impulses, as a result the insect loses its senses, becomes paralysed and eventually death occurs [26]. According to recent research, the DMT effect on central ganglia, subcuticular layer, and skeletal muscles was studied in salmon louse, which has shown tissue paralysis and apoptosis in the sensitised lice populations [13].

\section{Diisopropyl fluorophosphate (DFP) as an insecticide}

Diisopropyl fluorophosphate (DFP) also known as isoflurophate is a colourless liquid with the chemical formula C6H14FO3P and greasy texture. DFP are basically organophosphorus (phosphatidic esters of phosphoric acid) insecticidal compounds which play an effective role in the inhibition of certain enzymes of the central nervous system of almost all insects (including human lice), called as acetylcholinesterase enzymes of AChE. Such organophosphate based insecticides are being employed to eradicate human parasites, which kill the lice and do not let their eggs hatch [31].

\section{Mechanism of action of isoflurophate}

DFP is a highly neurotoxic agent used against a number of insect pests [32], in an experiment, $0.025 \mathrm{DFP}$ is a highly neurotoxic agent used against a number of insect pests [32], in an experiment, $0.025 \%$ of DFP was introduced in an antilouse lotion and louse obtained from an affected person was treated with it, after two weeks of observation it was noticed that no nits were hatched from the louse eggs [7]. These organophosphate (OP) insecticides tend to irreversibly halt the activation of $\mathrm{AChE}$ thus interfering in nerve transmission [33]. Usually, acetylcholinesterase (AChE) and choline are vital in controlling the physiological responses, as nerve conduction as well as membrane potential is mediated by the cholinergic system of the insects comprising of AChE, ACh, and ChA [34]. Acetylcholine (ACh) is hydrolysed by AChE, thus producing a number of neurosecretory molecules (including peptide products like allatotropins) for the nervous system signaling [35]. DFP and other OP compounds target the AChE enzyme and stop them from hydrolysing ChA, and that's how the neurotransmitters are not released, and the insect gets confused and unable to show any sort of response.

\section{Dimethicone: a potent remedy against Pediculus humanus}

Dimethicone/dimeticone also known as dimethylpolysiloxane or polydimethylsiloxane (PDMS), which belongs to polymeric organosilicon complexes, commonly referred as silicones, derived from synthetic silicon oil. Dimethicone is neither insecticide nor pediculicide but still it is an extremely impressive treatment for the removal of adult lice along with their nymphs/ eggs by physical means [1]. It has become one of the most demanding candidates to get rid of lice since its launch in the market in 2006. It was found in a study that 4Dimethicone/dimeticone also known as dimethylpolysiloxane or polydimethylsiloxane (PDMS), which belongs to polymeric organosilicon complexes, commonly referred as silicones, derived from synthetic silicon oil. Dimethicone is neither insecticide nor pediculicide but still it is an extremely impressive treatment for the removal of adult lice along with their nymphs/ eggs by physical means [1]. It has become one of the most demanding candidates to get rid of lice since its launch in the market in 2006. It was found in a study that $4 \%$ dimethicone lotion (or Hedrin 4\% dimeticone solution) treatment of head louse for fifteen minutes, abolished all motile stages and the appearance of louse from their eggshell [36]. Further, 70 to $75 \%$ of the affected individuals with Pediculus humanus capitis (head louse) pestilence were cured with good results, by the application of dimethicone in the UK [37]. 
Functioning strategies of PDMS in human louse PDMS mechanically greases the hair substratum by which louse and their nits become unable to stick with or fail to oviposition, by making a siliceous coating around the internal structures of louse through penetrating via tracheal openings or spiracles and thus suffocating the head louse by blocking the gaseous exchange system [38]. The impermeable blanket of dimeticone compound also stops water discharge out of the louse's body, thus disturbing homeostasis and leading to osmotic stress as well as a ceaseless immobility of its organs including gut or digestive system [39]. Furthermore, as the distal edge of a louse egg is not coated with the cement gland's secretions of the female louse, it is used for respiration and hence becomes a target site for the pediculicides and PDMS to get entered [5]. Albeit the silicone based products are hard to be washed off due to their nonvolatile properties [36], lice do not get a chance to retrieve and they remain paralysed till death.

\section{Significance of the newly introduced pediculicidal fusion:}

An ample usage of nonprescribed pediculicides also attributed as OTC, mostly in the developed countries leads to insensitivity of louse towards those particular treatments [1]. In the view of the fact that permethrin has been extensively utilised since last few decades as a successful pediculicide all over the world, which has been developed resistance in Pediculus humanus capitis due to occurrence of triple point mutation in the genes encoding certain amino acids [40]. Similarly, an astonishing resistance pattern has also been observed in human louse for the repetitive application of man made pyrethroids, due to mutation in the genes controlling sodium channels in louse (just like in common bed bugs) making them resistant to pyrethroids thus enabling them to survive easily [41]. By keeping all aforesaid experimental studies and their consequences, it can be estimated that an uncontrolled or excessive utilisation of pediculicidal products may give rise to resistance in these human ectoparasites instead of eliminating them permanently. Anyhow, our novel insight regarding the employment of an excellent, promising and viable merger of the aforenamed active antilouse ingredients by virtue of a pediculicidal tonic lotion vehicle. Surely, this amalgamation will provide us with a multipurpose product (containing reservoir of benefits of all above insecticidal agents) through lice killing, nit removal, paralysis, halted nerve transmission, loss of behavioural responses, inhibition of accessory glands' secretions and inability to oviposition of louse forever. Additionally, it will also reduce the mutational resisatnce in the genome of these insect parasites, decelerate the repeated use of them thus showing pacifying results after their use.

\section{Future Prospects}

Human lice being the undiscovered and poorly known members among various other insects of hexapoda are anticipiated to be explored in detail in order to find out the weaknesses of these vicious insects, which will be a plus point for all mankind. Due to unavailability of a well explained data and requirement of exactly the same physical conditions by these parasites (as temperature and humidity level) which are suitable for the humans as well, these barbaric insect species are on advantage since the evolution of Homo sapiens. We cannot dispose of these ectoparasites by washing our heads only but we should also have to take some wise steps. Fusion of the active pediculicidal candidates is being expected as a fruitful solution of this problem with a subsidised and operable policy on a global scale beacause it eliminates the threat of making these human parasites insensistive to the specific pediculicide by their usage, so that is why fresh combinations with an innovative thought and remedial impact against lice should be promoted.

\section{Concluding Remarks}

Coming to an end by the above discussion it can be apprehended that the perpetual clearance of the mulish blood feeders cannot be achieved by a single agent which may holds a confined working spectrum rather a multifunctioning remedy is needed. Our topical dogmatic review will undoubtedly provide an ideal proficiency against these human ectoparasites. Further studies are necessitated to explore the versatile treatments, able to the conditions less favourable for such insect species and to completely stop the reproduction mechanism in them.

\section{REFERENCES}

1. Asenov, A., Oliveira, F. A., Speare, R., Liesenfeld, O., Hengge, U. R., \& Heukelbach, J. (2010). Efficacy of chemical and botanical overthe counter pediculicides available in Brazil, and off label treatments, against head lice ex vivo. International journal of dermatology, 49(3), 324-330.

2. Speare, R., Canyon, D. V., \& Melrose, W. (2006). Quantification of blood intake of the head louse: Pediculus humanus capitis. International journal of dermatology, 45(5), 543-546.

3. Li, T., Liu, L., Zhang, L., \& Liu, N. (2014). Role of G-protein-coupled receptor-related genes in insecticide resistance of the mosquito, Culex quinquefasciatus. Scientific reports, 4(1), 1-9.

4. Dutra, J. M., Alves, A. D., Pessanha, T., Rachid, R., Souza, W. D., Linardi, P. M., ... \& Araujo, A. (2014). Prehistorical Pediculus humanus capitis infestation: quantitative data and low vacuum scanning microscopy. Revista do Instituto de Medicina Tropical de São Paulo, 56(2), 115-119.

5. Lapeere, H., Brochez, L., Verhaeghe, E., Vander Stichele, R. H., Remon, J. P., Lambert, J., \& Leybaert, L. (2014). Efficacy of products to remove eggs of Pediculus humanus capitis (Phthiraptera: Pediculidae) from the human 
hair. Journal of medical entomology, 51(2), 400407.

6. Priestley, C. M., Burgess, I. F., \& Williamson, E. M. (2006). Lethality of essential oil constituents towards the human louse, Pediculus humanus, and its eggs. Fitoterapia, 77(4), 303-309.

7. Bernstein, J. E. (1984). U.S. Patent No. 4,439,427 Washington, DC: U.S. Patent and Trademark Office.

8. Nouzova, M., Brockhoff, A., Mayoral, J. G., Goodwin, M., Meyerhof, W., \& Noriega, F. G. (2012). Functional characterization of an allatotropin receptor expressed in the corpora allata of mosquitoes. Peptides, 34(1), 201-208.

9. Krishnan, A., Almén, M. S., Fredriksson, R., \& Schiöth, H. B. (2014). Insights into the origin of nematode chemosensory GPCRs: putative orthologs of the Srw family are found across several phyla of protostomes. PloS one, 9(3), e93048.

10. Li, C., Song, X., Chen, X., Liu, X., Sang, M., Wu, W., .. \& Li, B. (2014). Identification and comparative analysis of $\mathrm{G}$ protein-coupled receptors in Pediculus humanus humanus. Genomics, 104(1), 58-67.

11. Gillott, C. (1996). Male insect accessory glands: functions and control of secretory activity. Invertebrate Reproduction \& Development, 30(1-3), 199-205.

12. Chen, P. S. (1984). The functional morphology and biochemistry of insect male accessory glands and their secretions. Annual review of entomology, 29(1), 233-255.

13. Bakke, M. J., Agusti, C., Bruusgaard, J. C., Sundaram, A. Y., \& Horsberg, T. E. (2018). Deltamethrin resistance in the salmon louse, Lepeophtheirus salmonis (Krøyer): Maternal inheritance and reduced apoptosis. Scientific reports, 8(1), 1-14.

14. Burkhart, C. N., \& Burkhart, C. G. (2005). Head lice: scientific assessment of the nit sheath with clinical ramifications and therapeutic options. Journal of the American Academy of Dermatology, 53(1), 129-133.

15. Duportets, L., Dufour, M. C., Couillaud, F. R. A. N. C. K., \& Gadenne, C. (1998). Biosynthetic activity of corpora allata, growth of sex accessory glands and mating in the male moth Agrotis ipsilon (Hufnagel). Journal of Experimental Biology, 201(16), 2425-2432.

16. Klowden, M. J. (2013). Physiological systems in insects. Academic press.

17. Fieber, L. A. (2017). The Oxford handbook of invertebrate neurobiology.

18. Konopova, B., Smykal, V., \& Jindra, M. (2011). Common and distinct roles of juvenile hormone signaling genes in metamorphosis of holometabolous and hemimetabolous insects. PloS one, 6(12), e28728.
19. Hauser, F., Cazzamali, G., Williamson, M., Park, Y., Li, B., Tanaka, Y., ... \& Grimmelikhuijzen, C. J. (2008). A genome-wide inventory of neurohormone GPCRs in the red flour beetle Tribolium castaneum. Frontiers in neuroendocrinology, 29(1), 142-165.

20. Weis, W. I., \& Kobilka, B. K. (2018). The molecular basis of $\mathrm{G}$ protein-coupled receptor activation. Annual review of biochemistry, 87, 897-919.

21. Parisi, F., Riccardo, S., Daniel, M., Saqcena, M., Kundu, N., Pession, A., ... \& Bellosta, P. (2011). Drosophila insulin and target of rapamycin (TOR) pathways regulate GSK3 beta activity to control Myc stability and determine Myc expression in vivo. BMC biology, 9(1), 1-13.

22. Kirkness, E. F., Haas, B. J., Sun, W., Braig, H. R., Perotti, M. A., Clark, J. M., ... \& Pittendrigh, B. R. (2010). Genome sequences of the human body louse and its primary endosymbiont provide insights into the permanent parasitic lifestyle. Proceedings of the National Academy of Sciences, 107(27), 12168-12173.

23. https: //www purdue.edu/newsroom/research/2010/100621HillL ice.html

24. Gainetdinov, R. R., Premont, R. T., Bohn, L. M., Lefkowitz, R. J., \& Caron, M. G. (2004). Desensitization of $G$ protein-coupled receptors and neuronal functions. Annu. Rev. Neurosci., 27, 107-144.

25. Khater, H. F., El-Shorbagy, M. M., \& Seddiek, S. A. (2014). Lousicidal efficacy of camphor oil, dphenothrin, and deltamethrin against the slender pigeon louse, Columbicola columbae. International Journal of Veterinary Science and Medicine, 2(1), 7-13.

26. Hossain, M. M., \& Richardson, J. R. (2011). Mechanism of pyrethroid pesticide-induced apoptosis: role of Calpain and the ER stress pathway. Toxicological Sciences, 122(2), 512525.

27. van der Wouden, J. C. et al. Interventions for treating head lice. The Cochrane Database of Systematic Reviews 2018 (2018).

28. 56. Kulikov, A. U. Determination of pyrethroid insecticide deltamethrin by micellar liquid chromatography with spectrophotometric detection. Chromatographia 66, 303-309 (2007).

29. Chrustek, A., Hołyńska-Iwan, I., Dziembowska, I., Bogusiewicz, J., Wróblewski, M., Cwynar, A., \& Olszewska-Słonina, D. (2018). Current research on the safety of pyrethroids used as insecticides. Medicina, 54(4), 61.

30. Johnson, J., Gooding, P. A., Wood, A. M., Taylor, P. J., Pratt, D., \& Tarrier, N. (2010). Resilience to suicidal ideation in psychosis: Positive selfappraisals buffer the impact of hopelessness. Behaviour research and therapy, 48(9), 883-889. 
31. Maunder, J. W. (1981). Novel phenomena arising from the use of acetylcholinesterase inhibiting insecticides against human head lice. Journal of Public Health, 3(1), 31-37.

32. Heiss, D. R., Zehnder, D. W., Jett, D. A., Platoff, G. E., Yeung, D. T., \& Brewer, B. N. (2016). Synthesis and storage stability of diisopropylfluorophosphate. Journal of chemistry, 2016.

33. Hamad, M. H., Adeel, A. A., Alhaboob, A. A. N., Ashri, A. M., \& Salih, M. A. (2016). Acute poisoning in a child following topical treatment of head lice (pediculosis capitis) with an organophosphate pesticide. Sudanese journal of paediatrics, 16(1), 63.

34. Colhoun, E. in Advances in insect physiology Vol. 1, 1-46 (Elsevier, 1963).

35. Lee, S. W., Kasai, S., Komagata, O., Kobayashi, M., Agui, N., Kono, Y., \& Tomita, T. (2007). Molecular characterization of two acetylcholinesterase cDNAs in Pediculus human lice. Journal of medical entomology, 44(1), 72-79.

36. Burgess, I. F. (2010). Do nit removal formulations and other treatments loosen head louse eggs and nits from hair?. Medical and Veterinary Entomology, 24(1), 55-61.

37. Kurt, Ö., Balcıŏlu, I. C., Burgess, I. F., Limoncu, M. E., Girginkardeşler, N., Tabak, T., ... \& Özbel,
Y. (2009). Treatment of head lice with dimeticone 4\% lotion: comparison of two formulations in a randomised controlled trial in rural Turkey. BMC Public Health, 9(1), 1-9.

38. Burgess, I. F. (2009). Current treatments for pediculosis capitis. Current Opinion in Infectious Diseases, 22(2), 131-136.

39. Balcığlu, İ. C., Karakuş, M., Arserim, S. K., Limoncu, M. E., Töz, S., Baştemur, S., ... \& Özbel, Y. (2015). Comparing the Efficacy of Commercially Available Insecticide and Dimeticone based Solutions on Head Lice, Pediculus capitis: in vitroTrials. Turkiye Parazitol Derg, 39(4), 305-9.

40. Brownell, N., Sunantaraporn, S., Phadungsaksawasdi, K., Seatamanoch, N., Kongdachalert, S., Phumee, A., \& Siriyasatien, P. (2020). Presence of the knockdown resistance (kdr) mutations in the head lice (Pediculus humanus capitis) collected from primary school children of Thailand. PLOS Neglected Tropical Diseases, 14(12), e0008955.

41. Yoon, K. S., Kwon, D. H., Strycharz, J. P., Hollingsworth, C. S., Lee, S. H., \& Clark, J. M. (2008). Biochemical and molecular analysis of deltamethrin resistance in the common bed bug (Hemiptera: Cimicidae). Journal of medical entomology, 45(6), 1092-1101. 